\title{
Heart-type fatty acid binding protein predicts cardiovascular events in patients with stable coronary artery disease: a prospective cohort study
}

\author{
Hui-Wen Zhang ${ }^{1}$, Jing-Lu Jin ${ }^{1}$, Ye-Xuan Cao ${ }^{1}$, Hui-Hui Liu ${ }^{1}$, Yan Zhang ${ }^{1}$, Yuan-Lin Guo ${ }^{1}$, Na-Qiong Wu ${ }^{1}$, \\ Cheng-Gang Zhu ${ }^{1}$, Ying Gao ${ }^{1}$, Rui-Xia Xu ${ }^{1}$, Qi Hua ${ }^{2}$, Yan-Fang Li ${ }^{3}$, Chuan-Jue Cui ${ }^{1}$, Geng Liu ${ }^{1}$, \\ Qian Dong ${ }^{1}$, Jing Sun ${ }^{1}$, Jian-Jun Li ${ }^{1}$
}

${ }^{1}$ State Key Laboratory of Cardiovascular Disease, Fu Wai Hospital, National Center for Cardiovascular Diseases, Chinese Academy of Medical Sciences and Peking Union Medical College, Beijing, China; ${ }^{2}$ Department of Cardiology, Xuanwu Hospital, Capital Medical University, Beijing, China; ${ }^{3}$ Department of Cardiology, Beijing Anzhen Hospital, Capital Medical University, Beijing, China

Contributions: (I) Conception and design: JJ Li; (II) Administrative support: JJ Li; (III) Provision of study materials or patients: JJ Li, YL Guo, NQ Wu, CG Zhu, Y Gao, Q Hua, YF Li; (IV) Collection and assembly of data: HW Zhang, JL Jin, YX Cao, HH Liu, Y Zhang, RX Xu, CJ Cui, G Liu, Q Dong, J Sun; (V) Data analysis and interpretation: HW Zhang, JL Jin, YX Cao; (VI) Manuscript writing: All authors; (VII) Final approval of manuscript: All authors.

Correspondence to: Prof. Jian-Jun Li, MD, PhD. State Key Laboratory of Cardiovascular Disease, Fu Wai Hospital, National Center for Cardiovascular Disease, Chinese Academy of Medical Sciences and Peking Union Medical College, Beijing 100037, China. Email: lijianjun938@126.com.

Background: Heart-type fatty acid binding protein (H-FABP) has been reported to be a prognostic predictor for cardiovascular outcome in acute coronary syndrome (ACS). However, its prognostic utility in patients with stable coronary artery disease (CAD) has not been well established. The aim of this study was to assess the association between H-FABP with the severity of coronary disease and cardiovascular events (CVEs) in patients with stable CAD.

Methods: A total of 4,370 angiography-proven $\mathrm{CAD}$ patients were consecutively enrolled. The severity of CAD was assessed by Gensini Score (GS) and the numbers of diseased vessels. The CVEs included cardiovascular death, myocardial infarction, stroke and coronary revascularization. Cox regression analysis with adjusted hazard ratios (HRs) and Kaplan-Meier analysis were used to evaluate the relation of H-FABP to CVEs in this cohort.

Results: During a median follow-up of 51 months, 353 CVEs occurred. Overall, patients in the highest levels of H-FABP group had increased rate of multi-vessel stenosis and higher GS compared with those in the lowest group $(\mathrm{P}<0.05$, respectively). Moreover, H-FABP levels were significantly higher in patients with events compared to those without $(\mathrm{P}<0.001)$. In Cox regression analysis, elevated H-FABP levels were found to be independently associated with a high risk of CVEs [adjusted HRs: 1.453; 95\% confidence intervals (CIs): 1.040-2.029, $\mathrm{P}=0.028$ ], especially with cardiovascular death (adjusted HRs: 2.865; 95\% CI: 1.315-6.243, $\mathrm{P}=0.008)$.

Conclusions: Our results demonstrated that H-FABP was also a useful predictor for CVEs in patients with stable CAD, which needed to be verified by further studies.

Keywords: Heart-type fatty acid binding protein (H-FABP); stable coronary artery disease; cardiovascular event (CVE)

Submitted Mar 13, 2020. Accepted for publication Aug 27, 2020.

doi: 10.21037/atm-20-2493

View this article at: http://dx.doi.org/10.21037/atm-20-2493 


\section{Introduction}

In cardiovascular medicine, biomarkers have been demonstrated to play multiple roles in understanding the pathophysiological complexity of coronary artery disease (CAD) and offering an integrated strategy to disease management. In fact, the exploration of biomarkers presenting acute myocardial injury is consistently a hot topic due to its relation to emergent status of CAD. In recent decades, several markers have been rapidly developed in the detection of myocardial injury, such as troponin I (TnI) or troponin $\mathrm{T}(\mathrm{Tn} \mathrm{T})$, and creatine kinase myocardial band (CK-MB), which have currently been used in the diagnosis of acute coronary syndrome (ACS). More interestingly, the usefulness of these markers for predicting cardiovascular events (CVEs) has clinically been of great interest (1-3).

Heart-type fatty acid binding protein (H-FABP), a cardiac biomarker released from cardiomyocytes, has previously been reported to be an early marker for acute myocardial injury $(2,4,5)$. Moreover, prior studies have also suggested a close relationship between H-FABP and atherosclerosis, acute pulmonary embolism, acute ischemic stroke and acute heart failure (6-10). Just recently, the clinical interest that applying it to the prognostic utility of CAD has been increasing $(5,11,12)$. For example, an early study in 2,287 patients with ACS revealed that $\mathrm{H}-\mathrm{FABP}$ was associated with increased risk of death and major cardiac events (13). Another study performed in 1,080 ACS patients with troponin-negative also indicated that H-FABP was additive to troponin in patients with suspected ACS (14). In addition, a recent study in 1,071 stable CAD patients with an average follow-up of 24 months has also showed a positive result of H-FABP in predicating CVE (15). However, these previous studies were limited by either small sample size or short-term follow-up. Therefore, further investigations may be needed to explore the prognostic utility of H-FABP in patients with stable CAD.

In the current study, we enrolled a consecutive, relatively large cohort with stable CAD to evaluate the association of baseline H-FABP levels with the disease severity and cardiovascular outcomes in angiography-proven patients with stable CAD.

We present the following article in accordance with the STROBE reporting checklist (available at http://dx.doi. org/10.21037/atm-20-2493).

\section{Methods}

\section{Study patients}

From January 2012 to February 2017, a total of 6,712 consecutive patients underwent coronary angiography (CAG) due to angina-like chest pain were enrolled in the three medical centers (Fuwai Hospital, National Center for Cardiovascular Diseases, Chinese Academy of Medical Sciences, Peking Union Medical College, Beijing, China; Department of Cardiology, Xuanwu Hospital, Capital Medical University, Beijing, China; Department of Cardiology, Beijing Anzhen Hospital, Capital Medical University, China). The baseline characteristics, angiographic features and laboratory data were collected from all patients. Patients without entire data, under the age of 18 years, with severe infectious or systematic inflammatory diseases, significant hematologic disorders, thyroid dysfunction, severe liver and renal insufficiency were excluded. Patients who did not conform the stable state of CAD according to the guideline were also excluded (3). Finally, 4,370 eligible patients with stable CAD were included. The study flowchart was shown as Figure 1.

The CAG was performed using standard clinical protocols with all angiograms interpreted by the experienced invasive cardiologists. The severity of CAD was evaluated by the numbers of lesion vessels and Gensini Score (GS) assessment system, which was defined according to stenosis severity as 1 -point for $<25 \%$ stenosis, 2 -point for $26-50 \%$ stenosis, 4-point for $51-75 \%$ stenosis, 8 -point for $76-90 \%$ stenosis and 32-point for total occlusion. The score was then multiplied by a multiplying factor that represented the importance of the lesion's position in the coronary arterial system. For example, 5 for the left main, 2.5 for the proximal left anterior descending or proximal left circumflex, 1.5 for the mid-segment and 1 for the distal segment.

The CVEs covered cardiovascular death, nonfatal MI, stroke, and coronary revascularization including percutaneous coronary intervention (PCI) and coronary artery bypass grafting (CABG). The methodology of follow-up was consistent with previous studies (16-18). In detail, after an initial appointment, all the patients were actively followed up at six months intervals by face-toface interviews and/or telephone connections with our well-trained cardiologists or nurses who were blinded to 


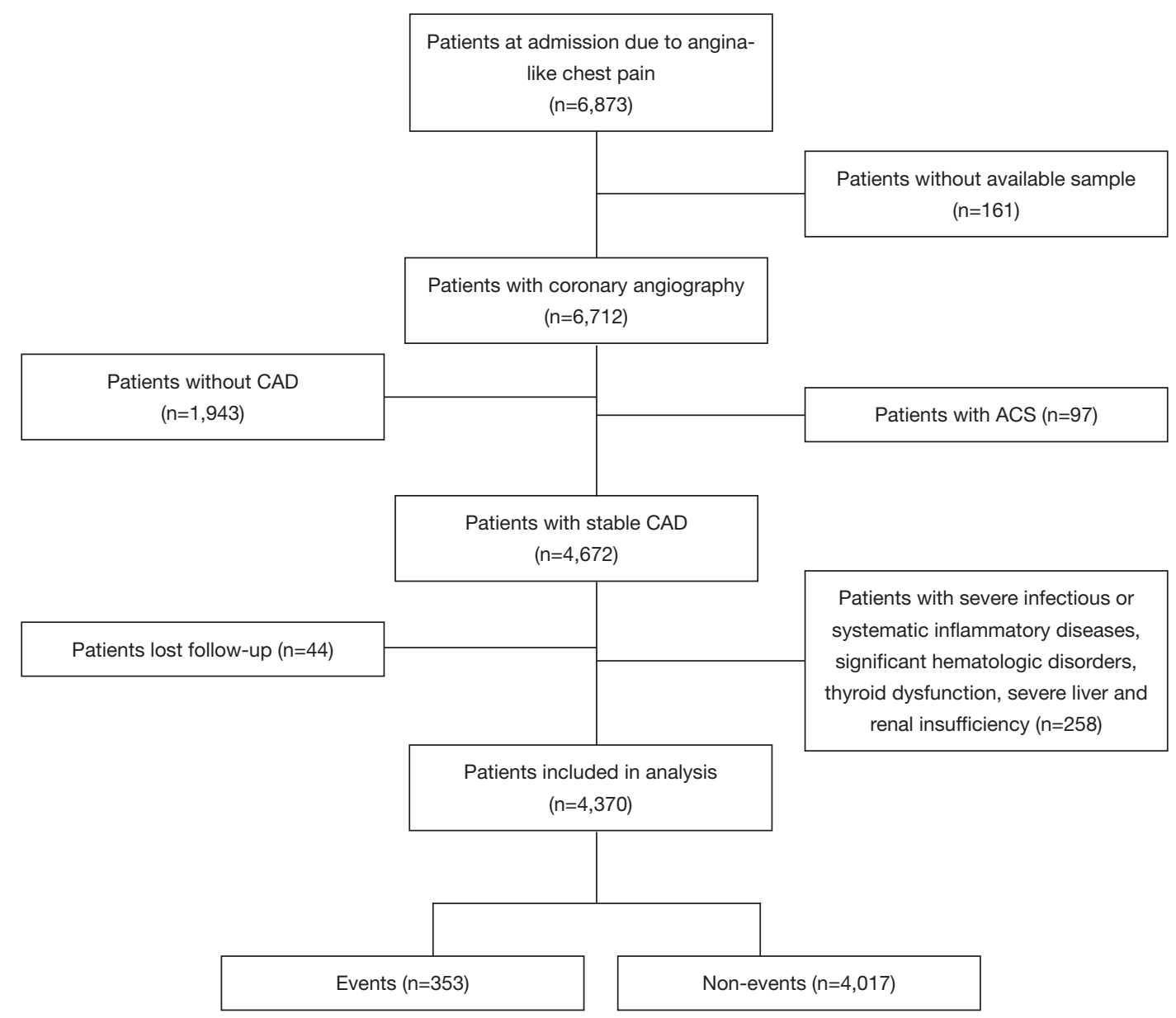

Figure 1 The study flowchart.

the purpose of this study. All available information from patients reported with possible event was collected. The death of a subject was reported by their relatives, the general practitioner, or the expert who treated the subject. Three experienced cardiologists who were blinded to this study classified the events independently.

The study was conducted in accordance with the Declaration of Helsinki (as revised in 2013). The study was approved by the hospital ethical review board of Fuwai Hospital and National Center for Cardiovascular Diseases, Beijing, China (No: 2013-442) and informed consent was taken from all the patients.

\section{Laboratory analysis}

The laboratory data were obtained from each patient with their venous blood samples taken after a 12 -hour overnight fasting. The lipid profiles were determined by automatic biochemistry analyzer (Hitachi 7150, Tokyo, Japan). In detail, the low-density lipoprotein cholesterol (LDL-C) concentration was analyzed by selective solubilization method (low-density lipid cholesterol test kit; Kyowa Medex, Tokyo). High-density lipoprotein cholesterol (HDL-C) concentration was measured by a homogeneous method (Determiner L HDL; Kyowa Medex, Tokyo). Total cholesterol (TC) and Triglyceride (TG) were determined by enzymatic methods. All other laboratory data mentioned were detected by standard haematological and biochemical tests. H-FABP was measured by Latex Immunoturbidimetric Method (Beijing Strong Biotechnologies, Inc. This turbidometric immunoassay was performed using the Hitachi 7180 chemistry analyzer). The reference interval of H-FABP was $<5 \mathrm{ng} / \mathrm{mL}$. The coefficient of variation for the assay was $<10 \%$. All the laboratory data were measured at baseline for each patient at the initial entry in hospital. 


\section{Statistical analysis}

The continuous data was expressed as mean \pm standard deviation (SD) or median (inter-quartile range) and the qualitative data were shown as frequencies or percentage. The categorical data were compared by Chi-square test. For determining differences among groups, a $t$-test was performed for the normally distributed data. As nonnormally distributed data, the Mann-Whitney $U$ test was performed for two-group comparisons and the KruskalWallis test for multi-group comparisons as appropriate. The event-free survival rates of total events and cardiovascular deaths among subgroups according to H-FABP levels were conducted by the Kaplan-Meier method and compared by the log-rank test. Cox regression hazard models were performed to calculate hazard ratios (HRs) and 95\% confidence intervals (CI) for CVEs in patients with different levels of H-FABP. All statistical analyses were performed using SPSS software version 23.0 (SPSS, Inc., Chicago, IL, USA).

\section{Results}

A total of 4,370 patients with stable CAD were finally included in this prospective multi-center study and the study flowchart was shown in Figure 1. The baseline characteristics of patients were provided in Table 1. The mean age of subjects was $58.2 \pm 9.7$ years and 3,087 patients (70.6\%) were male. H-FABP was measured in all 4,370 patients. The mean H-FABP level was $2.45 \mathrm{ng} / \mathrm{mL}$ in the total cohort. During a median of 51 months follow-up, 353 (8.1\%) patients occurred CVEs, included 69 cardiovascular deaths, 43 nonfatal MI, 90 stroke, and 151 coronary revascularization included PCI and CABG. Notably, serum H-FABP levels were significantly elevated in patients with events compared with ones without $(2.83 \pm 2.27 \mathrm{vs}$. $2.38 \pm 1.72 \mathrm{ng} / \mathrm{mL}, \mathrm{P}<0.001$, Table 1). Meanwhile, the patients with events were older and appeared to have higher rate of hypertension, higher level of hemoglobin A1c (HbA1c) when compared with those without events $(\mathrm{P}<0.05$, respectively, Table 1). Moreover, no significant difference of other laboratory data was found in patients with or without events $(\mathrm{P}>0.05$, respectively, Table 1$)$.

In addition, according to the quartiles of H-FABP levels, the subjects were divided into four subgroups as quartile $1(<1.50 \mathrm{ng} / \mathrm{mL})$, quartile $2(1.50-2.20 \mathrm{ng} / \mathrm{mL})$, quartile $3(2.21-3.00 \mathrm{ng} / \mathrm{mL})$, quartile $4(>3.0 \mathrm{ng} / \mathrm{mL})$. As shown in Table 2, the patients in the higher H-FABP group were older and had increased creatinine levels compared with ones in the lower H-FABP group $(\mathrm{P}<0.05$, respectively). Besides, the patients with higher H-FABP levels had higher rate of angiotensin converting enzyme inhibitors (ACEI)/angiotensin receptor blockers (ARB) medication $(\mathrm{P}<0.05)$, which might be related to the higher proportion of hypertension in these patients. Significantly, we found the association between H-FABP levels and risk of CVEs to follow a dose-response pattern with a higher risk among individuals in the fourth quartiles of H-FABP levels as compared with the first quartile $(5.8 \%$ vs. $7.4 \%$ vs. $8.3 \%$ vs. $11.3 \%, \mathrm{P}<0.001$, Table 2). The patients in the highest $\mathrm{H}$-FABP group showed near 1.5-fold higher rate of adverse events than ones in the lowest H-FABP group.

Then, we evaluated the relationship of H-FABP and the severity of coronary stenosis. The severity of CAD was assessed by GS and the numbers of lesion vessels. As shown in Table 2, the patients with elevated H-FABP levels had higher incidence of multiple vessels stenosis ( $\geq 3$ vessels, quartile 4 vs. quartile $1: 43.1 \%$ vs. $36.2 \%, \mathrm{P}=0.012$, Figure $2 A$ ). In addition, the patients in the highest quartile of H-FABP group showed significantly higher GS compared with ones in the lowest quartile of H-FABP group (mean GS: $37.9 \pm 35.7$ vs. $31.9 \pm 30.3, \mathrm{P}<0.001$, Table 2, Figure $2 B$ ).

We further explored the association between H-FABP levels with CVEs by stratifying the patients into 4 subgroups according to the quartile of H-FABP levels. As shown in Figure 3, the Kaplan-Meier curves demonstrated that the patients in the highest quartile of H-FABP were associated with the greatest risk of total cardiovascular outcomes (Figure $3 A$ ) and cardiovascular deaths (Figure 3B). Additionally, in Cox proportional-hazards model, elevated H-FABP levels were associated with increased risk of CVEs [unadjusted HR: 1.767; 95\% CIs: 1.301-2.400, $\mathrm{P}<0.001$, Figure $4 A]$. After adjusted for multiple valuables included age, gender, family history of CAD, dyslipidemia, hypertension, current smoking, diabetes and HbA1c, the patients in the upper levels of H-FABP remained to be independently correlated with worse cardiovascular outcomes (adjusted HR: 1.453; 95\% CIs: $1.040-2.029, \mathrm{P}=0.028$, Figure $4 B$ ). Notably, the patients with highest H-FABP levels showed a significantly increased risk of cardiovascular death (unadjusted HR: 4.427; 95\% CIs: 2.139-9.164, $\mathrm{P}<0.001$, Figure 4C), the results remained after adjusting for the established confounders (adjusted HR: 2.865; 95\% CIs: 1.315-6.243, $\mathrm{P}=0.008$, Figure $4 D$ ). 
Table 1 Baseline and clinical characteristics in study patients with and without events

\begin{tabular}{|c|c|c|c|c|}
\hline Variables & Total & Events & Non-events & $P$ value \\
\hline Patients & 4,370 & $353(8.1)$ & 4,017 (91.9) & \\
\hline Age (years) & $58.2 \pm 9.7$ & $59.9 \pm 10.2$ & $58.1 \pm 9.7$ & 0.001 \\
\hline Male & 3,087 (70.6) & $244(69.1)$ & $2,843(70.8)$ & 0.513 \\
\hline Hypertension & $2,866(65.6)$ & $257(72.8)$ & 2,609 (64.9) & 0.003 \\
\hline Dyslipidemia & 3,945 (90.3) & $312(88.4)$ & $3,633(90.4)$ & 0.211 \\
\hline Diabetes & $2,401(54.9)$ & $202(57.2)$ & $2,199(54.7)$ & 0.369 \\
\hline Current smokers & $1,345(30.8)$ & $88(24.9)$ & $1,257(31.3)$ & 0.013 \\
\hline Family history of CAD & $611(14.0)$ & $45(12.7)$ & $566(14.1)$ & 0.486 \\
\hline \multicolumn{5}{|l|}{ Laboratory data } \\
\hline Triglyceride (mmol/L) & $1.80 \pm 1.23$ & $1.83 \pm 1.25$ & $1.79 \pm 1.23$ & 0.585 \\
\hline $\mathrm{TC}(\mathrm{mmol} / \mathrm{L})$ & $4.14 \pm 1.17$ & $4.17 \pm 1.10$ & $4.13 \pm 1.18$ & 0.581 \\
\hline LDL (mmol/L) & $2.51 \pm 1.00$ & $2.51 \pm 0.95$ & $2.51 \pm 1.00$ & 0.937 \\
\hline $\mathrm{HbA1c}(\%)$ & $6.53 \pm 1.23$ & $6.85 \pm 1.32$ & $6.52 \pm 1.22$ & $<0.001$ \\
\hline H-FABP (ng/mL) & $2.45 \pm 1.86$ & $2.83 \pm 2.27$ & $2.38 \pm 1.72$ & $<0.001$ \\
\hline Tnl (ng/mL) & $0.004(0.002-0.009)$ & $0.004(0.002-0.010)$ & $0.004(0.002-0.009)$ & 0.275 \\
\hline ACEI or ARB & $2,024(46.3)$ & $174(49.3)$ & $1,850(46.1)$ & 0.242 \\
\hline
\end{tabular}

Values are expressed as the mean $\pm \mathrm{SD}$, the median with interquartile range or $\mathrm{n}(\%)$. CAD, coronary artery disease; BMI, Body mass index; TC, total cholesterol; LDL-C, low-density lipoprotein cholesterol; HbA1c, glycated hemoglobin; H-FABP, heart-fatty acid binding protein; Tnl, troponin I; ACEI, angiotensin converting enzyme inhibitors; ARB, angiotensin receptor blocker.

\section{Discussion}

In this multicenter, prospective study of 4,370 patients with stable $\mathrm{CAD}$, we investigated the diagnostic and prognostic values of H-FABP. The results freshly revealed the relation of H-FABP to stable CAD. Firstly, data defined that $\mathrm{H}-\mathrm{FABP}$ was associated with the severity of CAD in stable patients assessed by GS and the numbers of diseased vessels. Moreover, we found that H-FABP was an independent predictor for CVEs in this population. Importantly, sub- event analysis indicated that plasma H-FABP concentration was strongly associated with cardiovascular death. These findings were clinically novel and suggested the value of H-FABP in risk stratification among patients with stable $\mathrm{CAD}$, especially in predicting long-term mortality.

$\mathrm{H}-\mathrm{FABP}$ is a small cytoplasmic protein $(15 \mathrm{kDa})$ released from necrotic cardiac myocytes, which is highly specific to myocardium (19). It can reach the diagnostic concentration in blood just within 1 to 2 hours after the onset of clinical 
Table 2 Comparison of baseline and clinical characteristics of subjects according to the H-FABP levels (ng/mL)

\begin{tabular}{|c|c|c|c|c|c|}
\hline Variables & Quartile $1(<1.50)$ & Quartile 2 (1.50-2.20) & Quartile 3 (2.21-3.00) & Quartile 4 (>3.00) & $P$ value \\
\hline Patients & 1,134 & 1,091 & 1,147 & 998 & \\
\hline Age (years) & $54.2 \pm 9.0$ & $57.2 \pm 8.9$ & $59.0 \pm 9.3$ & $62.9 \pm 9.6$ & $<0.001$ \\
\hline Male & $798(70.4)$ & 781 (71.6) & $824(71.8)$ & $684(68.5)$ & 0.329 \\
\hline Hypertension & $703(62.0)$ & $678(62.1)$ & $752(65.6)$ & $733(73.4)$ & $<0.001$ \\
\hline Dyslipidemia & 1,016 (89.6) & $1,003(91.9)$ & $1,035(90.2)$ & $891(89.3)$ & 0.161 \\
\hline Diabetes & $602(53.1)$ & 599 (54.9) & $651(56.8)$ & $549(55.0)$ & 0.376 \\
\hline Current Smokers & $337(29.7)$ & $374(34.3)$ & 357 (31.1) & 277 (27.8) & 0.011 \\
\hline Family history of CAD & $172(15.2)$ & $179(16.4)$ & $154(13.4)$ & $106(10.6)$ & 0.001 \\
\hline \multicolumn{6}{|l|}{ Laboratory data } \\
\hline Triglyceride (mmol/L) & $1.85 \pm 1.43$ & $1.78 \pm 1.10$ & $1.79 \pm 1.12$ & $1.76 \pm 1.26$ & 0.403 \\
\hline $\mathrm{TC}(\mathrm{mmol} / \mathrm{L})$ & $4.14 \pm 1.23$ & $4.15 \pm 1.12$ & $4.14 \pm 1.20$ & $4.12 \pm 1.12$ & 0.923 \\
\hline LDL (mmol/L) & $2.52 \pm 1.06$ & $2.55 \pm 1.07$ & $2.49 \pm 0.91$ & $2.47 \pm 0.94$ & 0.231 \\
\hline HbA1c (\%) & $6.39 \pm 1.17$ & $6.47 \pm 1.23$ & $6.53 \pm 1.17$ & $6.78 \pm 1.31$ & $<0.001$ \\
\hline Troponin I (ng/mL) & $0.003(0.001-0.007)$ & $0.004(0.002-0.008)$ & $0.004(0.002-0.010)$ & $0.006(0.002-0.013)$ & $<0.001$ \\
\hline Creatinine $(\mu \mathrm{mol} / \mathrm{L})$ & $73.75 \pm 14.38$ & $75.40 \pm 13.59$ & $77.16 \pm 15.30$ & $85.65 \pm 22.34$ & $<0.001$ \\
\hline Multi-vessel disease $\geq 3$ & $412(36.2)$ & $436(40.1)$ & $472(41.3)$ & $430(43.1)$ & 0.012 \\
\hline Gensini score & $31.9 \pm 30.3$ & $33.8 \pm 31.2$ & $34.7 \pm 30.8$ & $37.9 \pm 35.7$ & $<0.001$ \\
\hline Cardiovascular events & $66(5.8)$ & $81(7.4)$ & $93(8.3)$ & $113(11.3)$ & $<0.001$ \\
\hline
\end{tabular}

Values are expressed as the mean $\pm \mathrm{SD}$, the median with interquartile range or $\mathrm{n}(\%)$. CAD, coronary artery disease; BMI, Body mass index; TC, total cholesterol; LDL-C, low-density lipoprotein cholesterol; HbA1c, glycated hemoglobin; H-FABP, heart-fatty acid binding protein; Tnl, troponin I; ACEI, angiotensin converting enzyme inhibitors; ARB, angiotensin receptor blocker.

symptoms $(4,20)$. Therefore, H-FABP is now recognized as an early cardiac biomarker of myocardial injury in patients with $\operatorname{ACS}(5,11,12)$. More interestingly, in addition to serving as a marker for myocardial injury, H-FABP is also reported to be a potential prognostic indicator for future cardiac outcomes. Recently, several studies have reported an association between elevated level of H-FABP and worse cardiac events in patients with ACS $(12-14,21,22)$. Therefore, its prognostic value in different status of cardiovascular disease may be of interest in cardiovascular medicine. However, only limited observations evaluated the role of $\mathrm{H}-\mathrm{FABP}$ in predicting the clinical outcomes 


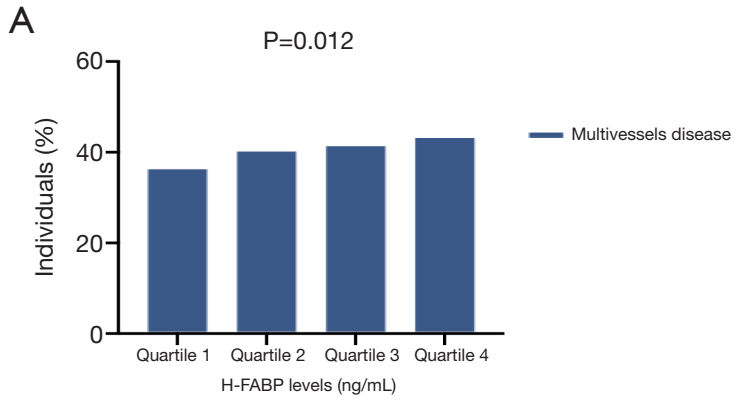

B

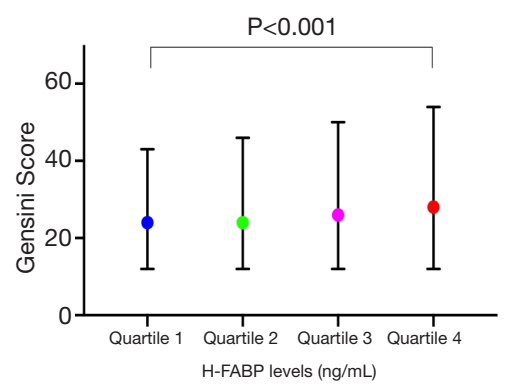

Figure 2 Association of heart fatty acids binding protein and the severity of coronary artery disease. Distribution of multivessels disease (A) and Gensini score (B) in different quartiles of heart fatty acids binding protein $(\mathrm{H}-\mathrm{FABP})$ levels.

in general population and stable CAD. For example, data from Takahata Study (23) in general Japanese population $(n=3,503)$ showed that a higher H-FABP level was associated with all-cause and cardiovascular deaths during a follow-up of 7 years. More recently, another study from Taiwan in 1,071 stable CAD patients with follow-up of 24 months showed that H-FABP was an independent predictor for CVE (15). Unfortunately, those studies appeared to be limited by either primary prevention or small sample size with short-term follow-up. Further study, hence, may be needed to clarify the role of H-FABP in patients stable CAD. That is a main reason why we carried out the present study.

The present study conducted in a larger Chinese cohort with up to 7.2 years follow-up, for the first time, showed that plasma H-FABP levels were significantly correlated with the severity of diseased coronary arteries. As we presented in the section of results, the degree of coronary artery stenosis was gradually aggravated in patients with elevated H-FABP levels. Such data provided the novel information regarding the role of $\mathrm{H}-\mathrm{FABP}$ in stable CAD. Moreover, whether H-FABP was related to the clinical

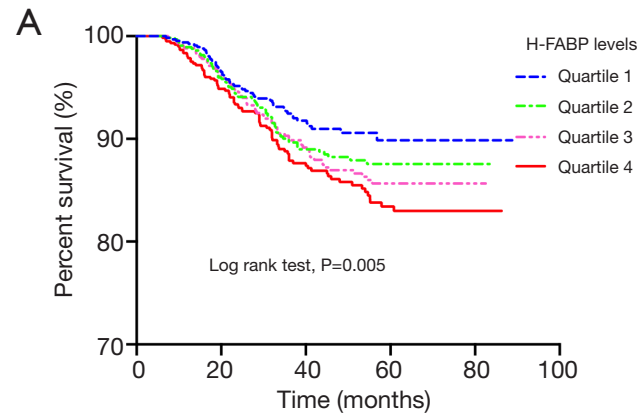

B

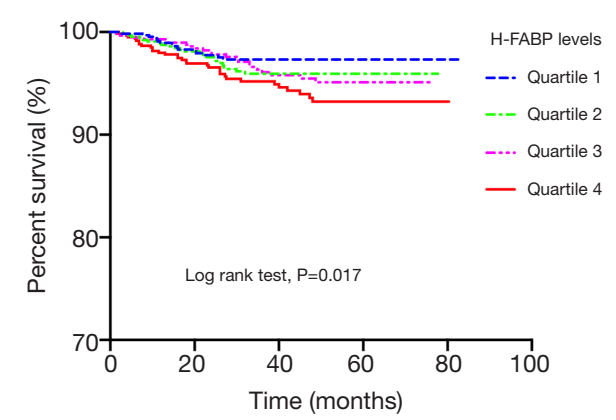

Figure 3 Survival analysis according to the heart fatty acids binding protein levels. Kaplan-Meier analysis of total cardiovascular events (A) and cardiovasular death (B) according to the quartiles of heart fatty acids binding protein (H-FABP).

outcomes in patients with stable CAD needed to be further studied.

To our knowledge, clinical outcomes are majorly dependent on the disease severity. The underlying mechanism regarding the impact of the disease severity on clinical outcomes in patients with CAD may be linked with the chances of the increased risk of coronary events. Since we have demonstrated that H-FABP was associated with the disease severity, we further explore the prognostic role of H-FABP in the present study. Obviously, our results showed that serum H-FABP levels were significantly elevated in patients with events compared with those without events, and the patients in the highest H-FABP group had near 1.5 -fold higher rate of adverse events than those in the lowest H-FABP group. Further analysis by Cox proportional-hazards model suggested that patients with highest H-FABP levels were independently associated with worse outcomes (adjusted HR: 1.453; 95\% CIs: 1.040 2.029, $\mathrm{P}=0.028$ ). Particularly, the H-FABP levels were significantly related to the risk of cardiovascular deaths even after adjusting for the established confounders (adjusted HR: 
A

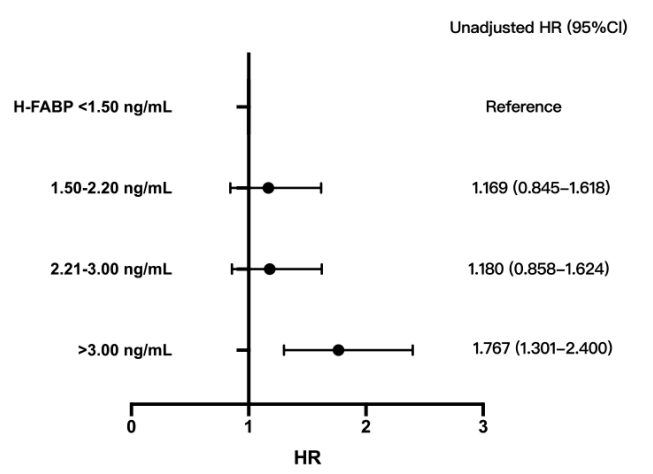

C

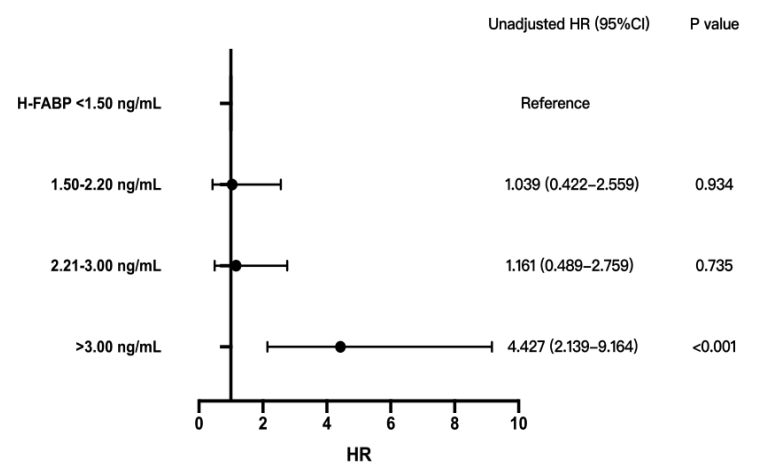

B

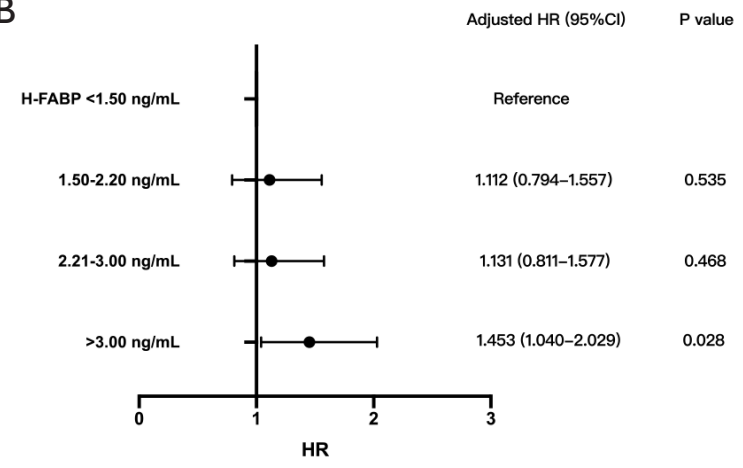

D

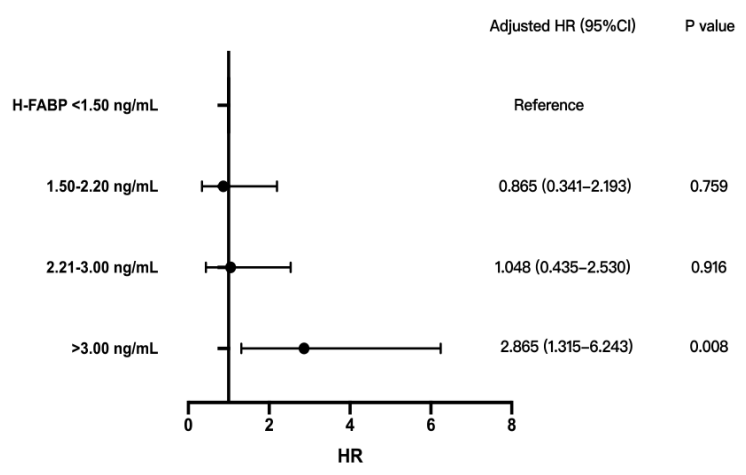

Figure 4 Hazard ratios for the association of heart fatty acids binding protein (H-FABP) with total cardiovascular events and cardiovascular death. Unadjusted model for cardiovascular events (A) and cardiovascular death (B) and adjusted model for cardiovascular events (C) and cardiovascular death (D) adjusted for age, gender, diabetes, dyslipidemia, hypertension, glycated hemoglobin, current smoking and family history of coronary artery disease. HR, hazard ratio; CI, confidence interval.

2.865; 95\% CIs: $1.315-6.243, \mathrm{P}=0.008)$. Although we could not confirm the exact mechanisms of the result that elevated levels of H-FABP were related to CVEs, the following items might be involved in the progression of CAD in subjects with elevated $\mathrm{H}$-FABP levels, such as that patients with higher H-FABP levels had more severe coronary disease, they were older and had higher prevalence of hypertension than those with lower H-FABP levels. Overall, the findings in the present study not only enhanced our understanding of the role of $\mathrm{H}-\mathrm{FABP}$ in patients with stable $\mathrm{CAD}$ but also provided hint for risk stratification in such population. Therefore, clinicians may need to pay more attention to the elevated levels of H-FABP in patients with stable CAD.

There were several potential limitations in the study. Firstly, as inherent to the nature of an observational and prospective study, only association but not causal link could be determined. In addition, H-FABP measured only at baseline and the levels might be affected by the medical intervention during the follow-up time. Finally, since the present study enrolled patients with normal creatinine clearance, we unable to determine whether H-FABP played a prognostic role in patients with renal dysfunction. Further studies were required to ascertain these findings.

In conclusion, the current study revealed a solid fact that H-FABP was a useful biomarker for worse outcomes due to its capability of predicting adverse clinical events in patients with stable $\mathrm{CAD}$, suggesting that $\mathrm{H}-\mathrm{FABP}$ might help to risk assessment in stable CAD.

\section{Acknowledgments}

Funding: This study was partly supported by Capital Health Development Fund (201614035) and CAMS Innovation Fund for Medical Sciences (2016-I2M-1-011), Fundamental 
Research Funds for the Central Universities (2018-F05) and Youth Research Fund of Peking Union Medical College (2018-XHQN03).

\section{Footnote}

Reporting Checklist: The authors have completed the STROBE reporting checklist, available at http://dx.doi. org/10.21037/atm-20-2493

Data Sharing Statement: Available at http://dx.doi. org/10.21037/atm-20-2493

Conflicts of Interest: All authors have completed the ICMJE uniform disclosure form (available at http://dx.doi. org/10.21037/atm-20-2493). The authors have no conflicts of interest to declare.

Ethical Statement: The authors are accountable for all aspects of the work in ensuring that questions related to the accuracy or integrity of any part of the work are appropriately investigated and resolved. The study was conducted in accordance with the Declaration of Helsinki (as revised in 2013). The study was approved by the hospital ethical review board of Fuwai Hospital and National Center for Cardiovascular Diseases, Beijing, China (No: 2013-442) and informed consent was taken from all the patients.

Open Access Statement: This is an Open Access article distributed in accordance with the Creative Commons Attribution-NonCommercial-NoDerivs 4.0 International License (CC BY-NC-ND 4.0), which permits the noncommercial replication and distribution of the article with the strict proviso that no changes or edits are made and the original work is properly cited (including links to both the formal publication through the relevant DOI and the license). See: https://creativecommons.org/licenses/by-nc-nd/4.0/.

\section{References}

1. Morrow DA, de Lemos JA. Benchmarks for the assessment of novel cardiovascular biomarkers. Circulation 2007;115:949-52.

2. McCann CJ, Glover BM, Menown IB, et al. Prognostic value of a multimarker approach for patients presenting to hospital with acute chest pain. Am J Cardiol 2009;103:22-8.

3. Thygesen K, Alpert JS, Jaffe AS, et al. Fourth Universal Definition of Myocardial Infarction (2018). Circulation 2018;138:e618-e651.

4. Kleine AH, Glatz JF, Van Nieuwenhoven FA, et al. Release of heart fatty acid-binding protein into plasma after acute myocardial infarction in man. Mol Cell Biochem 1992;116:155-62.

5. Pyati AK, Devaranavadagi BB, Sajjannar SL, et al. HeartType Fatty Acid Binding Protein: A Better Cardiac Biomarker than CK-MB and Myoglobin in the Early Diagnosis of Acute Myocardial Infarction. J Clin Diagn Res 2015;9:BC08-11.

6. Karbek B, Ozbek M, Bozkurt NC, et al. Heart-type fatty acid binding protein (H-FABP): relationship with arterial intima-media thickness and role as diagnostic marker for atherosclerosis in patients with impaired glucose metabolism. Cardiovasc Diabetol 2011;10:37.

7. Jo MS, Lee J, Kim SY, et al. Comparison between creatine kinase $\mathrm{MB}$, heart-type fatty acid-binding protein, and cardiac troponin $\mathrm{T}$ for detecting myocardial ischemic injury after cardiac surgery. Clin Chim Acta 2019;488:174-8.

8. Langer M, Forkmann M, Richter U, et al. Heart-type fatty acid-binding protein and myocardial creatine kinase enable rapid risk stratification in normotensive patients with pulmonary embolism. J Crit Care 2016;35:174-9.

9. Wunderlich MT, Hanhoff T, Goertler M, et al. Release of brain-type and heart-type fatty acid-binding proteins in serum after acute ischaemic stroke. J Neurol 2005;252:718-24.

10. Hoffmann U, Espeter F, Weiss C, et al. Ischemic biomarker heart-type fatty acid binding protein (hFABP) in acute heart failure - diagnostic and prognostic insights compared to NT-proBNP and troponin I. BMC Cardiovasc Disord 2015;15:50.

11. Liebetrau C, Nef HM, Dorr O, et al. Release kinetics of early ischaemic biomarkers in a clinical model of acute myocardial infarction. Heart 2014;100:652-7.

12. Ye XD, He Y, Wang S, et al. Heart-type fatty acid binding protein (H-FABP) as a biomarker for acute myocardial injury and long-term post-ischemic prognosis. Acta Pharmacol Sin 2018;39:1155-63.

13. O'Donoghue M, de Lemos JA, Morrow DA, et al. Prognostic utility of heart-type fatty acid binding protein in patients with acute coronary syndromes. Circulation 2006;114:550-7.

14. Viswanathan K, Kilcullen N, Morrell C, et al. Heart-type fatty acid-binding protein predicts long-term mortality and re-infarction in consecutive patients with suspected acute coronary syndrome who are troponin-negative. J Am Coll Cardiol 2010;55:2590-8.

15. Ho SK, Wu YW, Tseng WK, et al. The prognostic 
significance of heart-type fatty acid binding protein in patients with stable coronary heart disease. Sci Rep 2018;8:14410.

16. Jin JL, Sun D, Cao YX, et al. Triglyceride glucose and haemoglobin glycation index for predicting outcomes in diabetes patients with new-onset, stable coronary artery disease: a nested case-control study. Ann Med 2018;50:576-86.

17. Jin JL, Cao YX, Zhang HW, et al. Lipoprotein(a) and Cardiovascular Outcomes in Patients With Coronary Artery Disease and Prediabetes or Diabetes. Diabetes Care 2019;42:1312-8.

18. Liu HH, Cao YX, Li S, et al. Impacts of Prediabetes Mellitus Alone or Plus Hypertension on the Coronary Severity and Cardiovascular Outcomes. Hypertension 2018;71:1039-46.

19. Glatz JF and van der Vusse GJ. Cellular fatty acid-binding proteins: current concepts and future directions. Mol Cell

Cite this article as: Zhang HW, Jin JL, Cao YX, Liu HH, Zhang Y, Guo YL, Wu NQ, Zhu CG, Gao Y, Xu RX, Hua Q, Li YF, Cui CJ, Liu G, Dong Q, Sun J, Li JJ. Heart-type fatty acid binding protein predicts cardiovascular events in patients with stable coronary artery disease: a prospective cohort study. Ann Transl Med 2020;8(21):1349. doi: 10.21037/atm-20-2493
Biochem 1990;98:237-51.

20. Tanaka T, Hirota Y, Sohmiya K, et al. Serum and urinary human heart fatty acid-binding protein in acute myocardial infarction. Clin Biochem 1991;24:195-201.

21. Kilcullen N, Viswanathan K, Das R, et al. Heart-type fatty acid-binding protein predicts long-term mortality after acute coronary syndrome and identifies high-risk patients across the range of troponin values. J Am Coll Cardiol 2007;50:2061-7.

22. Matsumoto S, Nakatani D, Sakata Y, et al. Elevated serum heart-type fatty acid-binding protein in the convalescent stage predicts long-term outcome in patients surviving acute myocardial infarction. Circ J 2013;77:1026-32.

23. Otaki Y, Watanabe T, Takahashi H, et al. Association of heart-type fatty acid-binding protein with cardiovascular risk factors and all-cause mortality in the general population: the Takahata study. PLoS One 2014;9:e94834. 